\title{
HORMONE THERAPY IN TRANSSEXUALS AND THE RISK OF BREAST CANCER
}

Gabriela F. Araújo', Fábio F. Marques¹, Guilherme N. Corrêa', Mateus F. O. Vilela¹, Bráulio B. Rodrigues¹, Diogo T. de Lima', Mirian P. Silva'

${ }^{1}$ Centro Universitário de Anápolis (UniEVANGÉLICA) - Anápolis (GO), Brazil.

Objective: To carry out a systematic review of the literature on the relation of breast cancer in transsexual women using hormone therapy. Methods: Systematic review of the literature, using the PubMed databases, between 2003 and 2016 , using the key words "breast cancer", "femaile-to-male transsexuals", "male-to-female-transsexuals" "Hormone therapy ". From the total of 22 articles found, a total of 15 articles were selected in the last 13 years. Six studies were excluded because they were not the morphological alterations of the use of hormonal therapy in transsexuals, as well as the possibility and occurrences of breast cancer in these. Results: The nine articles selected show the relationship between hormone therapy and breast cancer in transsexuals, taking into account that in all the cases reported, the patients had firstdegree relatives with a history of breast, ovary or prostate cancer. However there are differences between studies. Lancet Oncol. 2013 September sees strong association of breast cancer laughter with serum concentration of testosterone. It discusses the action of testosterone to be aromatized in estrogen and stimulate the direct activation of estrogen receptors, exposed in two cases reported by Shao T, et al. in which in both the expression of estrogen receptors in transsexuals using testosterone presented in $90 \%$. While in a study by SLAGTER, Margarita H. et al. it is stated that testosterone promotes apoptotic effects in cancerous cells of breast cancer. Therefore, the simultaneous presence of high circulating testosterone and estrogens in these subjects could have prevented the biological action of E2, causing a histological image of involution. Conclusion: Although not yet studied, when referring to transsexuals from woman to man in use hormone therapy with high testosterone levels, strongly associated with positive family history, risks can not be excluded. As well as the presence of the BRCA2 mutation in transsexuals from man to woman using antiandrogen and estrogen may lead to breast cancer. A full family history is important in evaluating transgender people seeking hormone treatment. 